\title{
SONOLÊNCIA DIURNA EXCESSIVA PÓS-TRAUMATISMO DE CRÂNIO: ASSOCIAÇÃO COM MOVIMENTOS PERIÓDICOS DE PERNAS E DISTÚRBIO DE COMPORTAMENTO DO SONO REM
}

\section{Relato de caso}

\author{
Raimundo Nonato D. Rodrigues ${ }^{1}$, Aída A.A. Abreu e Silva²
}

\begin{abstract}
RESUMO - Um homem de 52 anos, procurou o Hospital. Universitário de Brasília com queixa de sono agitado. Sua esposa relatava, desde há cerca de 10 anos, intensa movimentação de membros e agressividade em meio a sonhos violentos. Desde então apresentava sonolência diurna excessiva. Havia relato de traumatismo de crânio há 34 anos e coma de 2 meses de duração. A vídeo-polissonografia revelou comportamento agressivo e agitado durante o sono REM, e movimentos periódicos de pernas. Havia importante sonolência diurna no teste de latências de sono. Foi instituído tratamento com levodopa-benzerazida 100/25 mg à noite. Após 10 semanas de evolução, houve melhora da movimentação noturna global, e desaparecimento dos episódios ligados a sonhos de conteúdo violento. Este caso nos permite analisar a associação entre trauma craniano e alterações nas vias dopaminérgicas (movimentos periódicos das pernas e distúrbio de comportamento do sono REM) e revisar a importância dos distúrbios na produção de hipocretina hipotalâmica na fisiopatologia desse quadro clínico.
\end{abstract}

PALAVRAS-CHAVE: traumatismo de crânio, vias dopaminérgicas, hipocretina, distúrbio de comportamento do sono REM, movimentos periódicos de pernas.

Excessive daytime sleepiness after traumatic brain injury: association with periodic limb movements and REM behavior disorder. Case report

\begin{abstract}
A 52 year-old male patient, had complaint of "restless sleep". His wife informed that for the past ten years the patient had presented intense and aggressive body movements, and sometimes, violent dreams. The patient also complained of excessive daytime sleepiness. His relevant previous medical history included a traumatic brain injury at the age of 28 which left him in coma for two months. A video-polysomnography showed periodic leg movements and, during REM sleep, aggressive and agitated behaviour. The multiple sleep latency test revealed extremely short latencies. Initially, he was treated with levodopa-benzerazide, 100/25 mg, 2 hours before bedtime. After 10 weeks his overnight behaviour pattern improved and leg movements diminished. This case supports the hypothesis of an association between cranial trauma and alterations in the dopaminergic pathways represented by periodic leg movements during sleep and a sleep behaviour disorder and proposes the possibility of hypothalamic hypocretin involvement in its pathophysiology.
\end{abstract}

KEY WORDS: cranial trauma, dopaminergic pathways, hypocretin, REM behaviour disorder, periodic leg movements.

Os traumatismos crânio-encefálicos (TCE) encontram-se entre a mais frequentes das afecções do sistema nervoso central (SNC). Trata-se de fenômeno sócio-econômico significativo e que atinge sobretudo indivíduos jovens ${ }^{1}$. O quadro sequelar, há muito reconhecido, é composto de cefaléias frequentes e sem sistematização precisa, resistentes aos analgésicos e associadas a vertigens e distúrbios neuro- psicológicos. Nos casos, mais graves responsáveis por comas prolongados, sequelas persistem em cerca de $60 \%$ dos pacientes após evolução de 3 a 5 anos $^{1}$. Os mecanismos lesionais são múltiplos, envolvendo rupturas axonais microscópicas, pequenas contusões hemorrágicas e sobretudo edema cerebral que retarda o retorno venoso. Além destes, deve-se mencionar as lesões hemorrágicas necróticas causadoras de

Laboratória de Sono do Hospital Universitário, Universidade de Brasília (UnB), Brasília DF, Brasil: ${ }^{1}$ Professor Assistente de Neurologia; ${ }^{2}$ Médica Residente.

Recebido 14 Novembro 2001, recebido na forma final 1 Fevereiro 2002. Aceito 15 Fevereiro 2002.

Dr. Raimundo Nonato D. Rodrigues - SQN 216 BI. A. Ap. 209 - 70875-010 Brasília DF - Brasil. 
edema cerebral, bem como a hipóxia, hipercapnia e congestão venosa as quais, favorecendo a diapedese nos tecidos atingidos, podem gerar hematomas intra-cerebrais secundários. A associação entre sonolência diurna excessiva e TCE foi proposta praticamente desde a descrição inicial da síndrome narcoléptica por Gelineau, no século XIX ${ }^{2}$ e tem sido confirmada em estudos ulteriores ${ }^{2,3}$. No entanto, a prevalência e os fatores de risco permanecem ainda incertos $^{4,5}$. Recentemente tem-se estudado o surgimento de perturbações nos padrões de sono, insônia e parassônias do despertar em intervalos de tempo variáveis após o TCE ${ }^{5,6}$. Por outro lado, a literatura mostra que a intercorrência de distúrbios comportamentais e movimentação involuntária de pernas em sono não é comum, e os possíveis mecanismos fisiopatológicos subjacentes ainda são desconheci$\operatorname{dos}^{7,8}$. Sabendo-se que o sono é uma atividade neurofisiológica ubíqua e de extrema importância para restaurar e revitalizar funções cognitivas como a memória e a atenção, pode-se estimar que o desenvolvimento de tais desordens poderá conferir pior prognóstico à recuperação do paciente vítima de TCE como também queda de sua qualidade de vida.

Assim, descrevemos o caso de um paciente que pela associação de traumatismo craniano, sonolência diurna excessiva, distúrbios motores e comportamentais do sono, nos apresentou interessantes questões do ponto de vista fisiopatológico e terapêutico.

\section{CASO}

Um Homen de 52 anos, uruguaio, tabagista (cachimbo), procurou o Ambulatório de Sono do Hospital Universitário de Brasília-DF, em 14 de dezembro de 2000, com queixas de sono agitado. Segundo relato da esposa, o paciente apresentava sonhos violentos e levantava da cama, gritava apavorado, gesticulava agressivamente, quase todas as noites. Contou que certa vez, sonhando que enfor-

Tabela 1. Comparação da distribuição percentual dos estágios de sono nas duas polissonografias realizadas.

\begin{tabular}{ccc}
\hline Estágio de sono & $\begin{array}{c}\text { \% TTS PSG } \\
09.03 .01\end{array}$ & $\begin{array}{c}\text { \% TTS PSG } \\
06.10 .01\end{array}$ \\
\hline 1 & 9,7 & 10,8 \\
2 & 38,5 & 39,6 \\
3 & 7,0 & 4,2 \\
4 & 9,4 & 22,4 \\
REM & 34,9 & 21,8 \\
\hline
\end{tabular}

PSG, polissonografia; TTS, tempo total de sono. cava um gato, havia tentado sufocar a esposa com um travesseiro. Desde então esta não mais dividia o leito com o marido. Tais sonhos e subsequente movimentação podiam ocorrer mesmo durante sestas diurnas. Esses episódios iniciaram-se há cerca de 10 anos. Desde essa época, reclamava também a esposa que o paciente apresentava sonolência excessiva durante o dia, chegando a dormir enquanto escrevia, assistia programas de televisão ou mesmo conversava ao telefone. Não havia, no entanto, qualquer queixa sugestiva de cataplexia.

Dentre seus antecedentes, notava-se o relato de um grave TCE há 34 anos, em acidente automobilístico, ao qual imediatamente seguiu-se coma de 2 meses de duração. Aparentemente, a partir daí, o paciente começou a apresentar certas peculiaridades de temperamento que $o$ impediram de permanecer no mesmo emprego durante muito tempo. Finalmente, havia relato de roncos pesados de vários anos de duração.

O exame clínico e neurológico revelava apenas pressão arterial de 160X100 mmHg. A sonolência diurna foi avaliada pela Escala de Sonolência de Epworth na qual o paciente obteve 16 pontos. $O$ teste de latência múltipla de sono (MSLT) mostrava sonolência diurna severa com latência média de sono de 2,7 minutos durante as cinco séries, com apenas um episódio de REM. A exploração funcional ventilatória e gasometria arterial em repouso respirando ar ambiente foram normais. Os resultados da avaliação polissonográfica estão relacionados nas Tabelas 1 e 2 . A saturação de hemoglobina foi $95 \%$. O registro em vídeo mostrou gritos, gesticulação e movimentação importante de pernas. Durante o último periodo REM, houve tentativa de levantar-se do leito.

Eletrencefalograma (EEG Nihon-Koden "Neurofax" de 18 canais) se mostrou sem anormalidades. Ressonância magnética mostrou áreas de encefalomalácia pós-traumática em região frontal e temporal esquerda, bem como no hemisfério cerebelar direito (Fig 1).

Com a hipótese diagnóstica de distúrbio de comportamento do sono REM, movimentos periódicos das pernas e síndrome da apnéia do sono leve, foi instituído tra-

Tabela 2. Comparação de parâmetros polissonográficos entre os dois exames realizados.

\begin{tabular}{lcc}
\hline & $\begin{array}{c}1{ }^{\circ} \text { EXAME: } \\
09.03 .01\end{array}$ & $\begin{array}{c}2^{\circ} \text { EXAME: } \\
06.10 .01\end{array}$ \\
\hline Latência de sono & $0,5 \mathrm{~min}$ & $20 \mathrm{~min}$ \\
Latência de sono lento & $10,5 \mathrm{~min}$ & $67,5 \mathrm{~min}$ \\
Latência de sono REM & $54 \mathrm{~min}$ & $136 \mathrm{~min}$ \\
IAH & 12,2 eventos $/ \mathrm{h}$ & 8,8 eventos $/ \mathrm{h}$ \\
Índice de movimento & & \\
periódicos de pernas & $105 \mathrm{mov} . / \mathrm{h}$ & $8,5 \mathrm{mov} . / \mathrm{h}$ \\
\hline
\end{tabular}

IAH, índice de apnéia-hipopnéia. 


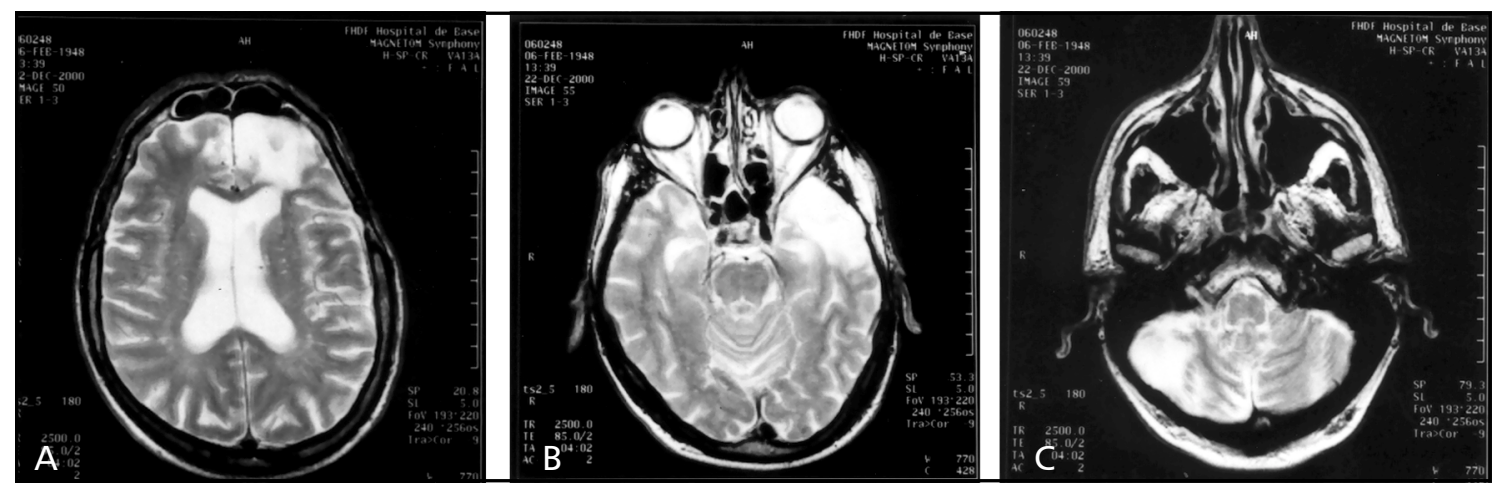

Fig 1. Ressonância magnética demonstrando áreas de encefalomalácia pós-traumática em região frontal (A) e temporal (B) esquerda bem como no hemisfério cerebelar direito (C).

tamento por levodopa-benzerazida 200/50 mg - 1/2 comprimido à noite. $\mathrm{O}$ paciente e a família relatam, após 10 semanas de evolução, melhora dos gritos e da movimentação noturna global e de membros, não tendo sido mais notado qualquer episódio ligado a sonho de conteúdo violento. Houve também melhora da sonolência diurna. Foi realizado controle polissonográfico cujos resultados serão discutidos mais adiante.

\section{DISCUSSÃO}

O tempo para o aparecimento dos distúrbios de sono em relação ao TCE pode variar. Alguns estudos observaram que pacientes após cinco anos do traumatismo costumam ter menos queixas de alterações de sono. Outros, no entanto, apontam casos surgidos até 29 anos após o trauma ${ }^{3}$. Guilleminault et al. ${ }^{2}$, em um dos primeiros estudos encontrados na literatura, arbitrariamente excluiram os pacientes com mais de 2 anos de traumatismo.

Alguns estudos sugerem predomínio de pessoas do sexo feminino ${ }^{6}$ e predisposição genética para o desenvolvimento dos distúrbios de sono, sobretudo a sonolência diurna excessiva9 ${ }^{9}$.

Aparentemente, os pacientes com TCE mais grave (coma mais prolongado) têm menos alterações de sono. Os autores atribuem tal ocorrência a uma subvalorização das queixas de sono pelos próprios doentes, o que poderia representar uma perda do insight $t^{5}$. Dentre as associações fenomenológicas possíveis dos distúrbios de sono pós-TCE, destacamse a dor e as desordens neurocomportamentais. A dor (cefaléia ou outras) e os distúrbios de sono representam uma associação mais importante no paciente pós-traumatizado de crânio com distúrbios neuropsicológicos do que nos pacientes neurológicos em geral $^{5}$. Tais alterações neuropsíquicas causariam prejuízo funcional cognitivo diurno e representariam segundo Guilleminault et al., uma "síndrome cere- bral orgânica" de grande importância e repercussão médico-legal ${ }^{2}$. Além disso, a fadiga é outra associação importante. Clinchot et $\mathrm{al}^{6}$ a encontraram em cerca de $80 \%$ de seus pacientes pós-traumatizados de crânio com distúrbios de sono. É de se imaginar que a fadiga também sirva de base para explicar a pseudo-hipersonia que alguns desses pacientes apresentam. Estes queixam-se de sonolência diurna, mas apresentam valores normais no MSLT. Alguns autores supõem a existência de lesões pós-traumáticas subjacentes em regiões talâmicas paramedianas, hipotalâmicas, subtalâmicas e mesmo mesencefálicas $^{9}$. Tais pacientes por vezes apresentam-se apáticos e com déficit de atenção.

No que se refere aos achados polissonográficos no pós-traumatismo de crânio, parece haver redução do tempo total de sono, do REM, dos grafoelementos do sono e do sono de ondas lentas, com aumento dos despertares. Tais alterações são mais encontradas nos pacientes com lesões no tronco cerebral, sobretudo na ponte. As alterações descritas apresentam semelhanças com as mudanças decorrentes da idade, o que levou alguns autores a supor que o traumatismo de crânio favoreça algum tipo de "envelhecimento" precoce dos padrões de sono ${ }^{6}$.

Dentre os distúrbios de sono mais importantes após $T C E$, encontramos a sonolência diurna excessiva ${ }^{10}$ acompanhada ou não de síndrome da apnéia obstrutiva de sono ${ }^{2,6}$. Esta última modalidade está particularmente associada a traumatismos raqui-medulares ("whiplash") $)^{2,9}$. Encontra-se também referência à insônia, à narcolepsia ${ }^{3}$ e à jactatio capitis ${ }^{6}$ como secundários à TCE. A dificuldade de iniciar o sono foi observada em $40 \%$ dos pacientes no estudo de Clinchot et $\mathrm{al}^{6}$. Beetar et $\mathrm{al}^{5}$ relatam que a insônia é queixa duas vezes mais comum em pacientes pós-traumatizados de crânio, que na população geral atribuindo-a a pos- 
síveis lesões na estrutura do tronco cerebral ou ainda à intercorrência de dor, fragmentando o sono.

A sonolência diurna excessiva associada ao TCE é fenômeno importante, ocorrendo em $25 \%$ da casuística de Clinchot et al., e concorre para o prejuízo funcional e perda da qualidade de vida dos pacientes $^{6}$ que se tornam susceptíveis à depressão reativa ${ }^{9}$. Em estudo recente, Guilleminault et al ${ }^{9}$ encontraram queixas e confirmaram distúrbios respiratórios do sono em cerca de $32 \%$ de seus pacientes com sonolência diurna pós-TCE. A maioria desses pacientes não apresentava obesidade (Indice de Massa Corporal $=26,6 \pm 1,8 \mathrm{Kg} / \mathrm{m}^{2}$ ). Por outro lado, $26 \%$ dos pacientes nesse estudo era portadora de algum tipo de anomalia da conformação crânio-facial que os predispunha ao distúrbio respiratório. Por outro lado, tem sido demonstrado experimentalmente o papel importante desempenhado pelas estruturas do sistema mesocorticolímbico de caráter dopaminérgico na modulação da sonolência diurna ${ }^{7}$. Dessa forma, sistemas dopaminérgicos hipoativos podem favorecer sonolência através de suas projeções para o córtex cerebral. Além disso, estudos em modelos animais sugerem que o peptídeo hipotalâmico hipocretina exerça influência sobre sítios colinérgicos e monoaminérgicos (dopaminérgicos) em vários níveis do SNC, e que seu déficit esteja diretamente ligado à desinibição do REM e à sonolência excessiva que caracterizam a narcolepsia?.

Ainda que esses estudos não tenham sido realizados especificamente em pacientes pós-TCE nem haja na literatura pertinente muitas referências objetivas, sabe-se que uma das consequências neurológicas do TCE pode ser o desenvolvimento progressivo de características clínicas parkinsonianas ${ }^{11}$, acompanhadas ou não de demência ${ }^{12}$. A doença de Parkinson, por sua vez, relaciona-se diretamente a déficit de dopamina na substância negra e envolve também certa hiperatividade colinérgica. Além disso, Arnulf et al. ${ }^{13}$ demonstraram em cerca de $36 \%$ de seus pacientes parkinsonianos com sonolência excessiva diurna, 2 ou mais episódios de REM precoce na MSLT (fenótipo narcolepsia-like). Assim, uma interessante questão com a qual nos defrontamos neste momento é a relação entre TCE e produção de hipocretina. Seria possível haver um déficit de hipocretina pós-trauma hipotalâmico? Isto nos parece uma hipótese ao menos tentadora, na medida em que explicaria não só a sonolência diurna excessiva como também implicaria a própria associação do TCE com distúrbios do sono associados à hipoatividade/desregulação do sistema dopaminérgico (dis- túrbio de comportamento do sono $\mathrm{REM}^{14}$ e movimentos periódicos de pernas - PLMS ${ }^{15}$ ) os quais foram a queixa de $27,2 \%$ dos pacientes estudados por Guilleminault et at. ${ }^{9}$.

De fato, Ripley et al. ${ }^{16}$, analisando os níveis de hipocretina no líquido cefalorraquidiano de pacientes portadores de diversas patologias neurológicas, encontraram redução na síndrome de Guillain-Barré, na hemorragia subaracnóidea espontânea e nas contusões cerebrais. Tais autores sugerem que esses dados podem compor o quadro de uma deficiência hipotalâmica mais extensa.

O estudo das relações entre o sistema dopaminérgico e os movimentos involuntários do sono em modelos caninos já demonstrou que neurônios dopaminérgicos mesencefálicos, especialmente os relacionados aos receptores $D_{2}$ e $D_{3}$, encontram-se envolvidos na regulação do sono e da cataplexia ${ }^{10,17}$. Da mesma forma, "movimentos de pernas" que lembram o PLMS foram recentemente descritos em cães narcolépticos ${ }^{17}$.

Em nosso paciente, notamos que as queixas de sonolência diurna excessiva foram tardias em relação ao trauma craniano (24 anos após), embora ainda dentro das expectativas segundo a literatura ${ }^{3}$. No entanto, bem antes dessa época, a família já reclamava de distúrbios de conduta, que foram responsáveis por constantes mudanças de emprego e mau relacionamento familiar e profissional. Talvez já se delineasse então a "síndrome cerebral orgânica" mencionada por Guilleminault et al, ${ }^{2}$. Infelizmente, não dispomos de testes neuropsicométricos que possam melhor avaliar essa hipótese.

O quadro clínico comportamental e a vídeopolissonografia confirmam o distúrbio de comportamento do sono REM, temporalmente associado a movimentos periódicos de pernas durante o sono. Diante do exposto acima, sugerimos que tal associação se faça através de desregulação ou desequilíbrio do sistema dopaminérgico decorrente do trauma craniano. A violência implícita num traumatismo que é responsável por coma de 2 meses de duração é clara e pode com sobras explicar danos ao tronco e hemisférios cerebrais. A RM atesta sequelas estruturais (lobo frontal, temporal e cerebelo). Tal mecanismo lesional parece-nos também suficiente para envolver as regiões hipotalâmicas e consequentemente afetar o metabolismo da hipocretina, como sugerido pela literatura ${ }^{16}$. Em resumo, o TCE seria responsável por alterações hipotalâmicas e do tronco cerebral, as quais afetariam tanto o metabolismo de hipocretina quanto o sistema dopaminérgico, o que por sua vez levaria ao 
surgimento dos distúrbios de movimentos involuntários de membros, alterações comportamentais durante o sono e sonolência diurna excessiva.

Revendo o tema das parassônias, Schenck e Mahowald 8 afirmaram que apesar de não ter sido relatado o distúrbio de comportamento do sono REM após trauma craniano, infecções, etc., havia evidências de que tais categorias não tardariam a ser implicadas. Um aspecto importante e ainda não elucidado na literatura é a eventualidade da participação dos circuitos dopaminérgicos na geração de sonhos patologicamente violentos que fazem parte do cortejo sintomatológico do distúrbio de comportamento do sono REM ${ }^{18}$.

Sob o ponto de vista terapêutico, a introdução de levodopa-benzerazida pareceu-nos a melhor opção, tendo em vista o papel importante desempenhado pelos PLMS nas queixas de sono agitado. Esperávamos com seu uso implementar a continuidade do sono e reduzir a sonolência diurna. Se abordássemos o caso pela vertente do distúrbio de comportamento do sono REM através do uso de clonazepam ${ }^{8}$, por exemplo, contribuiríamos para aumentar a sonolência diurna, possivelmente piorando o incipiente distúrbio respiratório confirmado à polissonografia.

A abordagem da síndrome da apnéia de sono através da utilização de CPAP (continuous positive airway pressure) nasal não nos pareceu conveniente, uma vez que não havia dessaturação de hemoglobina importante, o índice de perturbação respiratória era relativamente baixo (12,2 eventos/hora) e os episódios de perturbação ventilatória não eram significativamente associados aos movimentos de pernas, não explicando assim de maneira definida a sonolência diurna apresentada. Além disso, não vimos favoravelmente o uso de CPAP em paciente com movimentação corporal noturna tão violenta. Igualmente, nos casos de Guilleminault et al. há relato de melhora espontânea dos distúrbios respiratórios em número significativo de pacientes ${ }^{2}$.

Em consulta de retorno, cerca de 10 semanas após o início do uso de 100/25 mg de levodopabenzerazida à noite, havia melhora significativa da movimentação noturna. A esposa relatava que o leito do paciente não mais amanhecia desarrumado, ao mesmo tempo que havia maior grau de alerta durante o dia. Notamos também maior participação do paciente durante a consulta.

O exame polissonográfico de controle, realizado algumas semanas depois, mostrava aumento do sono delta e redução do REM em relação ao exame anterior (Tabela 1). Houve redução significativa do tempo passado em movimentos periódicos de pernas e do índice de perturbação respiratória (Tabela 2). A movimentação corporal registrada durante o REM foi normal e não houve qualquer comportamento violento associado ou não a sonhos durante toda a noite.

Concluímos que a boa resposta apresentada até agora com o implemento da atividade dopaminérgica neste paciente sugere o envolvimento destes sistemas na gênese da sonolência excessiva diurna e dos distúrbios de sono no paciente pós-traumatizado de crânio. Estudos bioquímicos e clínicos são necessários para melhor determinar a fisiopatologia dos fenômenos clinicamente observados, esclarecendo o papel desempenhado pela hipocretina, estabelecendo qual a real influência dos receptores dopaminérgicos $D_{2}$ e $D_{3}$ e ainda caracterizando o tipo e o envolvimento do dano hipotalâmico no pós-traumatismo de crânio.

\section{REFERÊNCIAS}

1. Aesch B, Jan M. Traumatismes cranioencéphaliques. Encycl Méd Chir Neurologie Paris: Elsevier, 1999:17585 A ${ }^{10}$.

2. Guilleminault C, Kym FF, Miles L, Van Den Hoed J. Posttraumatic excessive daytime sleepiness: a review of 20 patients. Neurology 1983; 33:1584-1589.

3. Lai J M, Castriotta RJ. Sleep disorders associated with traumatic brain injury. Sleep 1999;22:S 314.

4. Chiu FK, Wing YK, Lam LCW, et al. Sleep-related injury in the elderly: an epidemiological study in Hong-Kong. Sleep 2000;23:513-517.

5. Beetar JT, Guilmette TJ, Sparadeo FR. Sleep and pain complaintsin symptomatic traumatic brain injury and neurological populations. Arch Phys Med Rehab 1996;77:1298-1302.

6. Clinchot DM, Bogner J, Mysiw WJ, Fugate L, Corrigan J. Defining sleep disturbance after brain injury. Am J Phys Med Rehabil 1998;77:291-295.

7. Mignot E. Pathophysiology of narcolepsy In Kryger MH, Roth T, Dement WC (eds). Principles and practice of sleep medicine. 3.Ed. New York: Saunders, 2000:663-675.

8. Mahowald MW, Schenck CH. REM sleep parasomnias. In Kryger MH, Roth T, Dement WC (eds) Principles and practice of sleep medicine. 3.Ed. New York: Saunders, 2000:724-741.

9. Guilleminault C, Yuen KM, Gulevich BA, Karadeniz D, Leger D, Philip P. Hypersomnia after head-neck trauma. Neurology 2000;54:653-659.

10. Guilleminault C, Pelayo R. Idiopathic central nervous system hypersomnia. In Kryger MH, Roth T, Dement WC (eds). Principles and practice of sleep medicine. 3.Ed. New York: Saunders, 2000:687-692.

11. Hauser R, Zesiewicz. A doença de Parkinson: perguntas e respostas. $3^{\text {a }}$ edição. Atlas Medical Publishing. 2001:35-45.

12. Jordan BD. Chronic traumatic brain injury associated with boxing. Sem Neurol 2000;20:179-185.

13. Arnulf I, Kanofal E, Merino-Andreu M, et al. Narcolepsy-like phenotype in Parkinson's disease: how frequent in sleepy patients? Sleep 2001;24:A372.

14. Aldrich MS. Sleep disorders in dementias and related degenerative diseases. In Aldrich MS (ed). Sleep Medicine. New York: Oxford Univ Press. 1999:325-337.

15. Aldrich MS. Restless legs syndrome and periodic limb movement disorder. In Aldrich MS (ed) Sleep medicine. New York: Oxford Univ Press, 1999:175-185.

16. Ripley B, Overeem S, Fujiki N, et al. CSF hypocretin levels in various neurological conditions: low levels in narcolepsy and Guillain-Barré syndrome. Sleep 2001;24:A322.

17. Amitai N, Okura M, Fujiki N, et al. The dopaminergic system and sleep, and sleep-related involuntary movements. Sleep 2001; 24:A100.

18. Nielsen TA, Zadra A. Dreaming disorders. In Kryger MH, Roth T, Dement WC (eds). Principles and practice of sleep medicine. 3.Ed. New York: WB Saunders, 2000:753-772. 